\title{
Sagittal splitting of the temporalis muscle for encephalo-myo-synangiosis to prevent ischemic complications due to a swollen temporalis muscle without inhibiting collateral developments in patients with moyamoya disease
}

\author{
Toshio Machida, MD, ${ }^{1,2}$ Yoshinori Higuchi, MD, ${ }^{3}$ Shigeki Nakano, MD, ${ }^{1,2}$ Masaki Izumi, MD, ${ }^{1}$ \\ Satoshi Ishige, MD, ${ }^{1}$ Atsushi Fujikawa, MD, ${ }^{4}$ Yuichi Akaogi, MD, ${ }^{5}$ Junichiro Shimada, MD, ${ }^{5}$ and \\ Junichi Ono, MD \\ 'Department of Neurosurgery, Chiba Cerebral and Cardiovascular Center; ${ }^{2}$ Department of Neurosurgery, Eastern Chiba \\ Medical Center; ${ }^{3}$ Department of Neurological Surgery, Chiba University Graduate School of Medicine; ${ }^{4}$ Department of \\ Neurosurgery, Chibaken Saiseikai Narashino Hospital; and ${ }^{5}$ Department of Neurology, Chiba Cerebral and Cardiovascular \\ Center, Chiba, Japan
}

OBJECTIVE Encephalo-myo-synangiosis (EMS) is an effective revascularization procedure for the treatment of moyamoya disease (MMD). However, the temporalis muscle used for EMS sometimes swells and causes ischemic complications by compressing the underlying brain. This study aimed to elucidate the effect of sagittal splitting (SS) of the muscle for prevention of ischemic complications and its impact on the postoperative development of collateral vessels.

METHODS In this historical case-control study, we analyzed 60 hemispheres in adult patients with MMD who underwent EMS using the temporalis muscle from December 1998 to November 2017. The muscle was divided anteroposteriorly by coronal splitting, and the anterior, posterior, or both parts of the muscle were used for EMS in 17, 4, and 39 hemispheres, respectively. In cases performed after 2006, the muscle was halved by SS, and the medial half was used for EMS to reduce the muscle volume $(n=47)$. The degree of postoperative muscle swelling was evaluated by measuring the maximum thickness of the muscle on CT scans obtained 3 to 7 days after surgery. The collateral developments of the anterior deep temporal artery (aDTA), posterior deep temporal artery (pDTA), and middle temporal artery (MTA) were assessed using digital subtraction angiography and MR angiography performed 6 months or more after surgery. RESULTS SS significantly reduced the temporalis muscle thickness from $12.1 \pm 5.0 \mathrm{~mm}$ to $7.1 \pm 3.0 \mathrm{~mm}(p<0.01)$. Neurological deterioration due to the swollen temporalis muscle developed in 4 of the 13 hemispheres without SS (cerebral infarction in 1, reversible neurological deficit in 2, and convulsion in 1) but in none with SS. There were no significant differences in the postoperative collateral developments of the aDTA, pDTA, and MTA between hemispheres with and without SS. The MTA more frequently developed in hemispheres with EMS in which the posterior part of the muscle was used $(30 / 37)$ than those in which this part was not used $(4 / 16)(p<0.01)$.

CONCLUSIONS SS of the temporalis muscle might prevent neurological deterioration caused by the swollen temporalis muscle by reducing its volume without inhibiting the development of the collateral vessels.

https://thejns.org/doi/abs/10.3171/2018.1.JNS172244

KEYWORDS encephalo-myo-synangiosis; moyamoya disease; sagittal splitting; swollen temporalis muscle; vascular disorders 
$\mathrm{M}$ oyamoya disease (MMD) is a steno-occlusive cerebrovascular disease. It is characterized by progressive occlusion of the terminal portion of the internal carotid artery and proliferation of the collateral vessels (moyamoya vessels). ${ }^{14}$ Both ischemic and hemorrhagic stroke may develop due to impaired hemodynamics or disruption of the fragile moyamoya vessels. Revascularization surgeries, such as superficial temporal artery to middle cerebral artery (STA-MCA) anastomosis and encephalo-duro-arterio-synangiosis, are therapeutic options for the treatment of MMD that increase cerebral blood flow, resolve moyamoya vessels, and prevent future strokes. ${ }^{14}$ Encephalo-myo-synangiosis (EMS) is a revascularization surgery for MMD in which the temporalis muscle is used as a donor organ. ${ }^{10}$ Because the muscle covers a large area of the brain, EMS widely improves impaired hemodynamics. ${ }^{8}$ However, despite its efficacy, the inserted muscle for EMS sometimes swells, compresses the underlying brain, and causes neurological deterioration (Fig. 1). ${ }^{6,19}$ The ischemic brain of MMD patients is vulnerable to focal increases in pressure; thus, prevention of mechanical compression by the inserted muscle is mandatory.

Sagittal splitting (SS) and coronal splitting (CS) of the temporalis muscle are modified techniques that have been applied in the fields of craniomaxillofacial and skull base surgery. ${ }^{3}$ In patients with MMD, the efficacy of the technique for EMS on the reduction of muscle volume has been reported. ${ }^{12}$ However, the impact of the modified technique on surgical results is not certain. This study aimed to evaluate the efficacy of SS of the temporalis muscle for prevention of complications and its impact on collateral development after surgery.

\section{Methods}

\section{Study Design}

This retrospective case-control study was approved by the institutional review board at our hospital. From December 1998 to November 2017, a total of 69 hemispheres in 54 consecutive patients with adult MMD underwent EMS with or without STA-MCA anastomosis at our institution and were analyzed.

\section{Surgical Indication}

Patients with a symptomatic cerebral hemisphere were considered candidates for revascularization. Since 2015, patients with asymptomatic hemisphere and hemorrhagic manifestation have also been considered candidates according to the results of the Japanese Adult Moyamoya trial. ${ }^{16}$

\section{Surgical Procedures}

In all patients, a combined direct and indirect bypass (STA-MCA anastomosis with EMS) was planned.

In 39 hemispheres, a large fronto-temporo-parietal craniotomy was performed (Table 1). A curved skin incision that originated from the forehead to the base of the mastoid process was made (Fig. 2A). The temporalis muscle was dissected from the calvaria (Fig. 2B), cut vertically to introduce the STA to the anastomosis point on the brain surface (coronal splitting; Fig. 2C), and divided into 2 parts (anterior and posterior). After fronto-temporo-parietal craniotomy and dural incision, STA-MCA anastomosis was performed in an end-to-side manner. Both the anterior and posterior parts of the temporalis muscle were sheeted over the brain and sutured to the dura (EMS; Fig. 2D). The bone flap was drilled off at the site of the temporalis muscle insertion to avoid compression of the muscle (Fig. 2C) and was fixed to the skull with titanium plates. After epidural and/or subcutaneous drainage tube placement, the skin was closed. From 1998 to 2006, EMS was performed in 9 hemispheres in this manner. Since 2006, we have been splitting the temporalis muscle in the sagittal plane to reduce its volume $(n=30)$. The temporalis muscle was halved in the sagittal plane by use of electric cautery or scissors (Fig. $2 \mathrm{E})$. Then, only the medial part of the muscle was seated over the brain surface (Fig. 2F). After cranioplasty (Fig. $2 \mathrm{G}$ ), the remaining lateral halves of the muscle were seated over the bone flap (Fig. 2H). In addition, to make a space for the inserted muscle, the bone flap was drilled off of its inner layer and was fixed to the skull with a little gap on the inserted muscle. The epidural and/or subcutaneous drainage tube was removed the next day after surgery in cases performed before 2006. In cases performed since 2006, the tube was retained for 3 days.
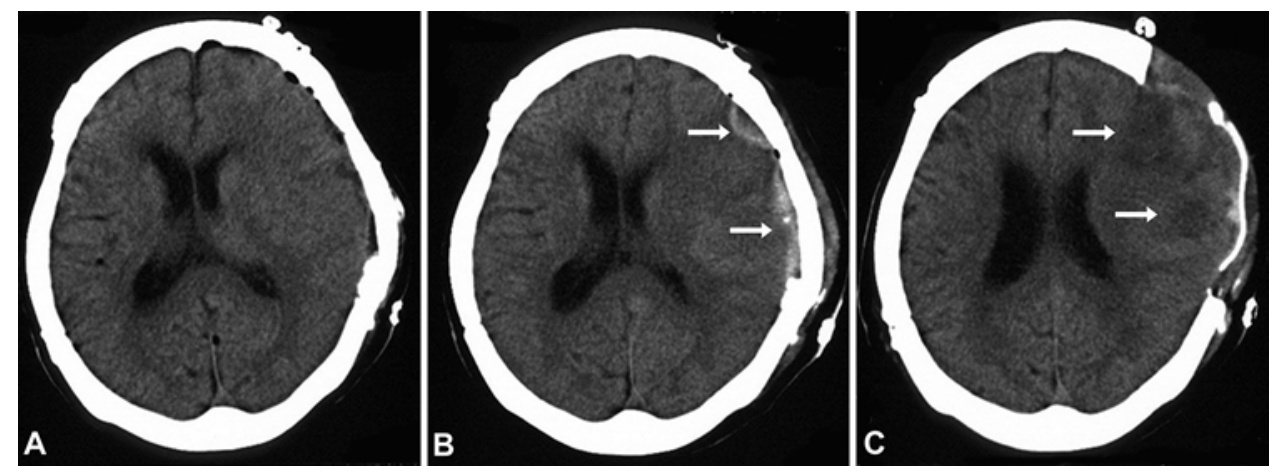

FIG. 1. Representative case of ischemic stroke due to the swollen temporalis muscle. A: Axial CT scan obtained 1 day after surgery, demonstrating no lesion in the operated left cerebral hemisphere. B: Three days after the surgery, the inserted temporalis muscle swelled and compressed the underlying brain (arrows). C: A decompressive craniotomy was performed, but cerebral infarction developed just below the temporalis muscle (arrows). 
A
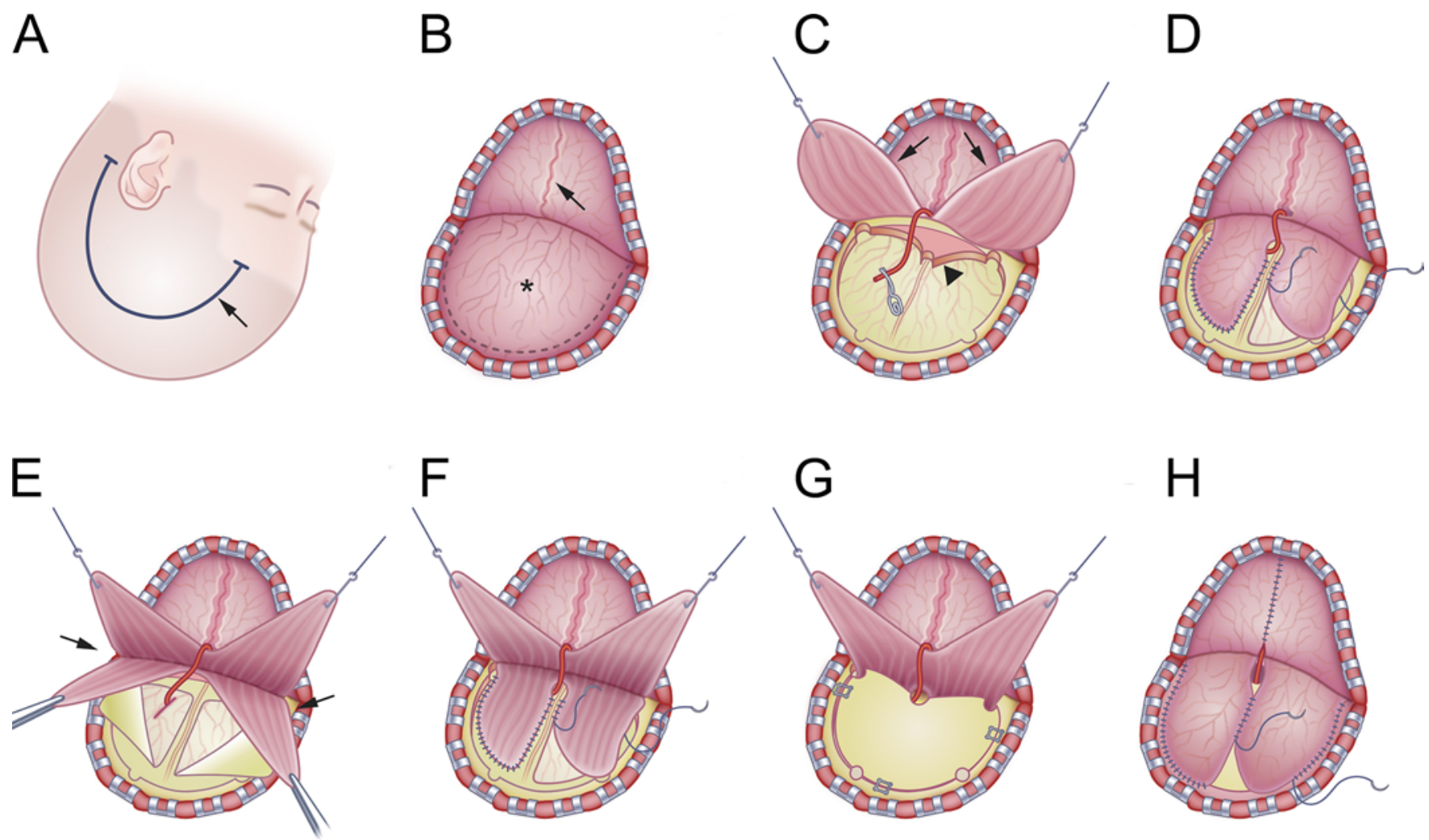
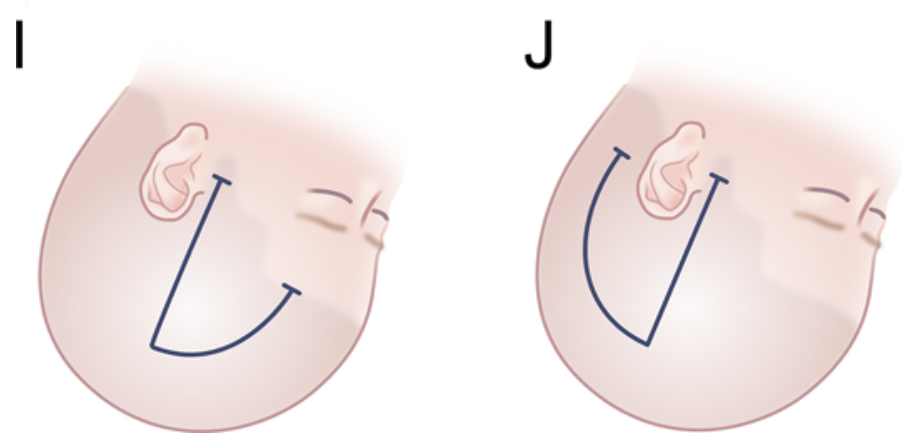

FIG. 2. Coronal and sagittal splitting of the temporalis muscle. A: Skin incision (arrow). B: The parietal branch of the STA (arrow) is harvested, and the entire temporalis muscle (asterisk) is exposed. C: Coronal splitting of the temporalis muscle (arrow). The bone is drilled off at the site of the temporalis muscle insertion (arrowhead). D: Both the anterior and posterior portions of the muscle are seated over the brain surface and sutured to the dura. E: Sagittal splitting of the temporalis muscle (arrows). F: The medial halves of the anterior and posterior portions of the temporalis muscle are seated over the brain surface and sutured to the dura. G: Cranioplasty is performed. $\mathrm{H}$ : The lateral halves of the temporalis muscle cover the bone flap. I: Skin incision of the anteriorhalf craniotomy. J: Skin incision of the posterior-half craniotomy. Copyright Toshio Machida. Published with permission. Figure is available in color online only.

In 21 hemispheres, only the anterior-half (Fig. 2I) or the posterior-half (Fig. 2J) skin incision and half craniotomy were performed in order to harvest the parietal branch of the STA (pSTA) and avoid injury of the naturally developed collateral vessels of the dura mater. The skin incision was made just above the pSTA that curved anteriorly or posteriorly. Consequently, only the anterior $(n=17)$ or the posterior $(n=4)$ half of the temporalis muscle was used for EMS. SS of the temporalis muscle was applied in 13 of 17 and 4 of 4 hemispheres with anterior- and posterior-half craniotomies, respectively (Table 1).
In the remaining 9 hemispheres, SS was not performed in any part of the muscle as the muscle was too thin, and the hemispheres were thus excluded from this analysis.

\section{Evaluation of Temporalis Muscle Swelling and Collateral Developments}

The degree of temporalis muscle swelling was evaluated by measuring the maximum thickness of the muscle using axial CT scans obtained 3 to 7 days after surgery.

Collateral developments were evaluated using digital 


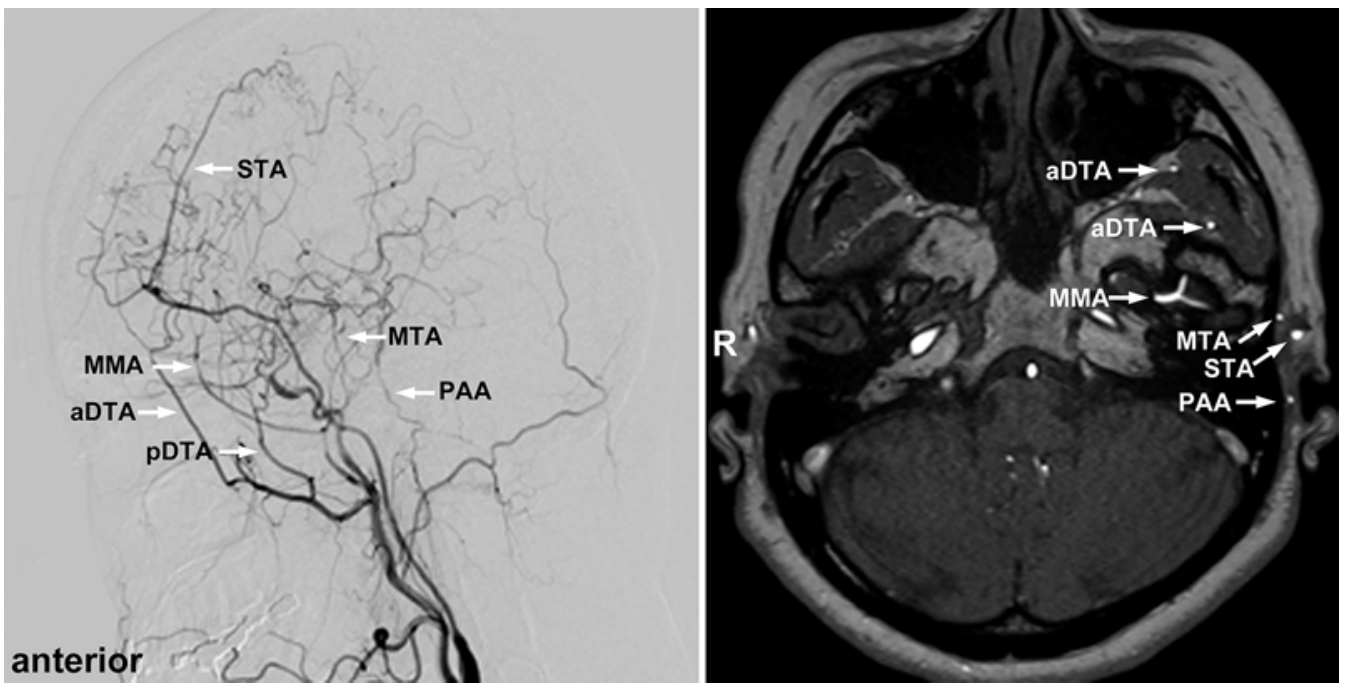

FIG. 3. Collateral development after EMS. Lateral cerebral angiogram (left) and axial MR angiogram obtained at the level of the zygomatic arch (right), demonstrating the collateral developments of the aDTA, pDTA, MTA, STA, MMA, and posterior auricular artery (PAA).

subtraction angiography and/or MR angiography using a 3-T system (Magnetom Skyra, Siemens AG, Healthcare Sector) performed at least 6 months after surgery (Fig. $3)$. The developments of the anterior deep temporal artery (aDTA), posterior deep temporal artery (pDTA), and middle temporal artery (MTA), the major branches of the external carotid artery supplying blood to the temporalis muscle, were assessed. The developments of the STA and the middle meningeal artery (MMA) were also evaluated.

\section{Anatomical Distributions of the Collateral Vessels}

Because the potential collateral vessels may be injured by CS, we studied the anatomical distributions of aDTA, pDTA, and MTA in relation to pSTA. Using axial MR angiography, we measured the distances between the pSTA and the aDTA, pDTA, and MTA, at the level of the zygomatic arch in hemispheres where these collateral arteries developed. The course of the MTA was as follows: 1) anteriorly, 2) posterosuperiorly (Fig. 4 left), and 3) superiorly (Fig. 4 right).

\section{Definition of Neurological Deterioration by the Swollen Temporalis Muscle}

MMD patients who undergo revascularization surgery often experience transient neurological deterioration that mimics neurological deterioration due to the swollen temporalis muscle..$^{5,15,17}$ Therefore, we defined this complication as neurological deterioration with radiological evidence of the substantially thickened temporalis muscle compressing the underlying brain on CT scanning.

\section{Clinical Follow-Up}

Patients were followed up 3 months, 6 months, and every year after surgery, and recurrent stroke was recorded.

\section{Statistical Analysis}

The Mann-Whitney U-test or Student t-test was performed for continuous variables. The chi-square test or Fisher's exact test was performed for categorical variables. Risk of recurrent stroke was determined using the KaplanMeier method with comparisons made using log-rank sta-
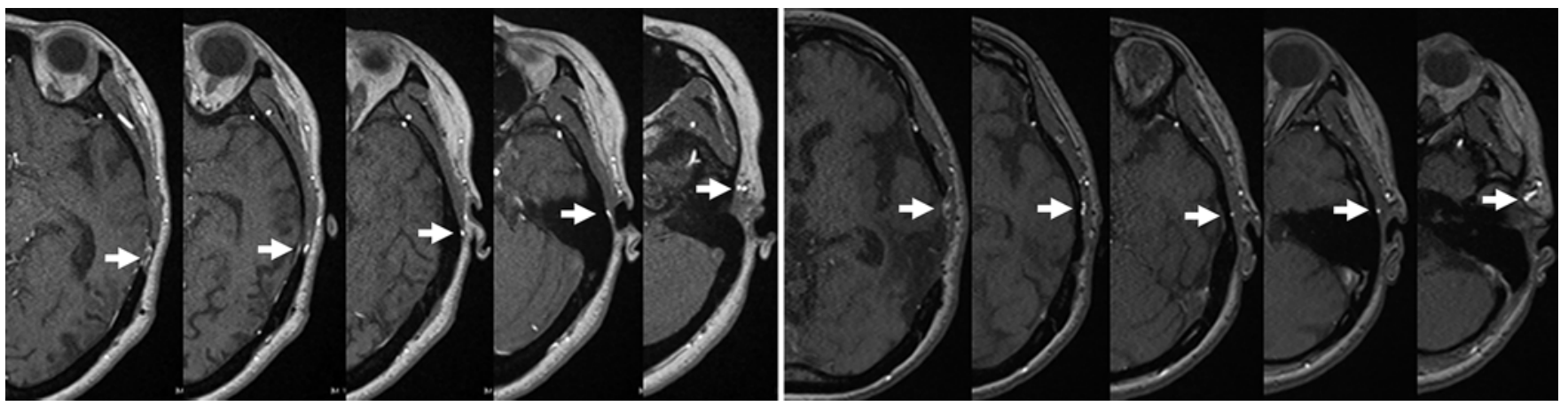

FIG. 4. Axial MR angiograms showing the course of the MTA. The MTA runs posteriorly (left, arrows) and superiorly (right, arrows) after the branching from the STA. 
TABLE 1. Characteristics of the 60 hemispheres

\begin{tabular}{lccc}
\hline & w/o SS $(n=13)$ & w/ SS $(n=47)$ & p Value \\
\hline Median age (range), yrs & $52(28-65)$ & $41(18-74)$ & $<0.05$ \\
\hline Sex & & & NS \\
\hline Female & 11 & 33 & \\
\hline Male & 2 & 14 & \\
\hline Initial presentation & & & NS \\
\hline$\quad$ Ischemia & 11 & 37 & \\
\hline Hemorrhage & 2 & 10 & \\
\hline Suzuki stage & & & NS \\
\hline III & 10 & 34 & \\
\hline IV & 2 & 12 & \\
\hline VI & 1 & 1 & \\
\hline Part of the temporalis & & & \\
muscle used for EMS & & 30 & \\
\hline$\quad$ Whole & 9 & 13 & \\
\hline Anterior & 4 & 4 & \\
\hline Posterior & 0 & & \\
\hline
\end{tabular}

NS = not significant.

tistics. All comparisons were planned, and the tests were 2-tailed; $\mathrm{p}<0.05$ was considered statistically significant. Analyses were performed using EZR software.?

\section{Results}

Table 1 demonstrates the characteristics of the study population. Patients in whom SS was performed were significantly younger. There were no significant differences between the groups in terms of sex, initial presentation of the disease, MMD stage, and the part of the temporalis muscle used for EMS. Postoperative muscle thickness was decreased by SS from $12.1 \pm 5.0 \mathrm{~mm}$ to $7.1 \pm 3.0 \mathrm{~mm}(\mathrm{p}$ $<0.01$, Table 2). Neurological deterioration due to a swollen temporalis muscle developed in 4 hemispheres without SS and in no hemispheres with SS (p < 0.01). Details of the 4 hemispheres with complications are shown in Table 3. Cerebral infarction, reversible neurological deficit, and convulsion developed in 1,2, and 1 hemispheres, respectively. Additional decompressive craniotomy was required in 2 hemispheres. Cerebral infarction developed in one of these 2 hemispheres, and the patient's modified Rankin Scale (mRS) score worsened from 1 to 2 . During the follow-up period (mean $5.47 \pm 3.95$ years) 0 and 3 recurrent strokes developed in hemispheres without and with SS, respectively. The recurrence rates were calculated as $0 \%$ and $1.52 \%$ /patient-year for each; this difference was not statistically significant (Table 2).

Postoperative collateral developments were studied in 12 of 13 and 41 of 47 hemispheres without and with SS, respectively (Table 4). The aDTA, pDTA, and MTA developed equally irrespective of SS within the same usage of the muscle (whole or anterior part). The MTA developed more frequently (30/37) in hemispheres with EMS in which the posterior part of the muscle was used than in those in which it was not used (4/16), and the difference was significant $(\mathrm{p}<0.01)$.
TABLE 2. Surgical results

\begin{tabular}{lccc}
\hline & $\begin{array}{c}\text { w/o SS } \\
(\mathrm{n}=13)\end{array}$ & $\begin{array}{c}\text { w/ SS } \\
(\mathrm{n}=47)\end{array}$ & $\begin{array}{c}\mathrm{p} \\
\text { Value }\end{array}$ \\
\hline $\begin{array}{l}\text { Mean max muscle thickness (SD), } \\
\mathrm{mm}\end{array}$ & $12.1(5.0)$ & $7.1(3.0)$ & $<0.01$ \\
$\begin{array}{l}\text { Neurological deterioration due to } \\
\text { swollen temporalis muscle, } \mathrm{n}(\%)\end{array}$ & $4(21 \%)$ & $0(0 \%)$ & $<0.01$ \\
\hline Mean FU period (SD), yrs & $9.77(2.84)$ & $4.19(3.22)$ & $<0.01$ \\
\hline Recurrent stroke & 0 & 3 & \\
\hline Recurrence rate (\%/patient-year) & 0 & 1.52 & $\mathrm{NS}^{*}$ \\
\hline $\begin{array}{l}\text { FU = follow-up. } \\
\text { * Log-rank test. }\end{array}$ & & & \\
\end{tabular}

Table 5 demonstrates the anatomical relationship between the pSTA and the aDTA, pDTA, and MTA. At the level of the zygomatic arch, the aDTA, pDTA, and MTA were located $42.0-60.4 \mathrm{~mm}, 20.8-48.3 \mathrm{~mm}$, and -13.5 to $7.8 \mathrm{~mm}$ anterior to the pSTA, respectively. The MTA ran posterosuperiorly (20/34) or superiorly (14/34), but no artery ran anteriorly after branching from the STA (Table 6).

\section{Discussion}

In this study, we demonstrated that SS of the temporalis muscle reduces its volume, which might prevent neurological deterioration by the swollen muscle without affecting the collateral developments.

\section{Effect of Sagittal Splitting for the Prevention of Complications}

Because the hemodynamically impaired brain of MMD patients is vulnerable to increases in intracranial pressure, mechanical compression due to the inserted muscle can easily cause focal ischemia in the underlying brain. ${ }^{12}$ Takemura et al. first reported 3 cases of the complication, wherein ischemia developed several days after the surgery just below the inserted muscle. ${ }^{19}$ They proposed the significance of depressurization below the muscle. Thinning of the bone flap and postoperative usage of a hyperosmotic agent prevented the complication. Fujimura et al. reported a complication in which revision of the EMS was required. ${ }^{6}$ Because the inserted muscle was wedged by bone flap, they speculated that venous congestion due to muscle compression was the cause of the swelling. A wide bone window at the site of the temporalis muscle insertion resolved the complication. Thus, this complication has been identified in some case reports; however, the incidence of the complication is unclear.

The postoperative stroke rate of revascularization surgery for MMD has been reported to vary from $0 \%$ to $33.3 \%$ according to the evidence. ${ }^{7,11}$ Patient age, initial manifestation, stability of the disease, and surgical procedures will affect the surgical result. Regarding the combined bypass surgery for adult MMD, postoperative stroke rates have been reported to range from $7.9 \%$ to $15.3 \%$, similar to the findings in the current study. ${ }^{2,411}$ Because the details of stroke in these studies are unclear, it is uncertain whether some of the complications were due to the swollen tempo- 
TABLE 3. Details of the 4 hemispheres with neurological deterioration due to swollen temporalis muscle

\begin{tabular}{clccccc}
\hline Age (yrs), Sex & Initial Presentation & Muscle Thickness & Neurological Deterioration & DC & Preop mRS Score & Outcome/mRS Score \\
\hline $49, \mathrm{~F}$ & TIA & $9.9 \mathrm{~mm}$ & Infarction & Yes & 1 & Dysphasia/2 \\
\hline $63, \mathrm{~F}$ & Infarction & $23 \mathrm{~mm}$ & RND & Yes & 1 & Full recovery/1 \\
\hline $52, \mathrm{~F}$ & Hemorrhage/infarction & $14.5 \mathrm{~mm}$ & RND & No & 1 & Full recovery/1 \\
\hline $47, \mathrm{~F}$ & TIA & $13.0 \mathrm{~mm}$ & Convulsion & No & 1 & Full recovery/1 \\
\hline
\end{tabular}

$D C=$ decompressive craniotomy; RND = reversible neurological deficit; $T I A=$ transient ischemic attack.

ralis muscle or whether countermeasures against muscle swelling were undertaken. In this study, neurological deterioration due to the swollen temporalis muscle developed in 4 of the $13(21 \%)$ hemispheres without SS. Patients in 3 of these 4 cases were spared permanent neurological deficits by intensive care; however, cerebral infarction developed in 1 hemisphere despite additional decompressive craniotomy, and the patient experienced dysphasia. Because revascularization surgery for MMD is performed to prevent future stroke, it is mandatory to minimize perioperative neurological deterioration. We then took countermeasures against the swollen temporalis muscle as follows: 1) reduced the muscle volume by SS, 2) drilled off the inner layer of the bone flap and fixed it to the skull with a small gap above the muscle, and 3) continued postoperative drainage for 3 days. Consequently, the postoperative maximum thickness of the muscle decreased from $12.1 \mathrm{~mm}$ to $7.1 \mathrm{~mm}$, and the complication did not develop thereafter.

\section{Impact of SS and CS on Collateral Developments}

SS prevented ischemic complications by reducing muscle volume. However, because only the medial half of the muscle was used for EMS (Fig. 2C), the reduction of collateral developments of EMS with the modification was also a concern. In this study, we demonstrated that the collateral developments from the aDTA, pDTA, and MTA were not affected by SS (Table 4).

Given the vascularity and large volume of the temporalis muscle, it has been used as a pedicled flap in the reconstruc-

TABLE 4. Collateral development after revascularization

\begin{tabular}{cccc}
\hline & \multicolumn{3}{c}{ No. of Hemispheres (\%) } \\
\cline { 2 - 4 } & Whole & Anterior & Posterior \\
\hline w/o SS & 8 & 4 & \\
\hline aDTA & $1(13)$ & $3(75)$ & \\
\hline pDTA & $6(75)$ & $4(100)$ & \\
\hline MTA & $7(88)$ & $2(50)$ & 4 \\
\hline STA & $5(63)$ & $3(75)$ & $0(0)$ \\
\hline MMA & $6(75)$ & $4(100)$ & $1(25)$ \\
\hline W/ SS & 25 & 12 & $3(75)$ \\
\hline aDTA & $11(42)$ & $3(25)$ & $4(100)$ \\
\hline PDTA & $24(88)$ & $11(92)$ & $4(100)$ \\
\hline MTA & $20(81)$ & $2(17)$ & \\
\hline STA & $22(88)$ & $12(100)$ & $5(42)$ \\
\hline MMA & $20(77)$ & &
\end{tabular}

tion surgery of the craniomaxillofacial and skull base region. ${ }^{3,18}$ Various splitting techniques, including SS and CS, have been applied based on muscle fiber configuration, and the course of the supplying vessels and the vascular anatomy of the muscle have been well investigated in these fields.

The temporalis muscle is supplied mainly by 3 branches of the external carotid artery: aDTA, pDTA, and MTA. The aDTA and pDTA originate from the maxillary artery at the infratemporal fossa and run superiorly. The aDTA and pDTA supply blood to the anterior $21 \%$ and middle $41 \%$ of the temporalis muscle, respectively. ${ }^{3}$ Because these arteries are located on the medial surface of the muscle, they are preserved in the medial half of the muscle by SS. ${ }^{3,18}$ This anatomical characteristic accounts for comparable developments of the aDTA and pDTA in hemispheres with and without SS in this study.

The MTA, another main branch supplying the temporalis muscle, originates from the STA below the posterior edge of the zygomatic arch. It runs posteriorly or parallel to the STA and divides into 2 branches: the fascial (fMTA) and muscular (mMTA) branches. The fMTA transverses the zygomatic arch and supplies blood to fatty tissue and deep temporal fascia. The mMTA is located on the medial surface of the temporalis muscle similar to the DTAs and supplies blood to the posterior 38\% of the muscle. Thus, the mMTA is preserved in the medial half of the muscle by SS, whereas the fMTA remains in the lateral half of the muscle that is seated over the skull. Regarding the hemispheres in which the entire temporalis muscle was used in EMS $(n=33$; Table 4$)$, because there was no significant difference in the collateral development of the MTA in hemispheres with and without SS of the entire temporalis muscle (7 of 8 and 20 of 25 , respectively), we think that the collateral development originated from the mMTA, which would have been preserved in the medial half of the muscle.

We also demonstrated that the MTA developed more frequently in hemispheres with EMS using the posterior part of the temporalis muscle (30 of 37) than in those in which the posterior part was not used (4 of 16; Table 4).

TABLE 5. Distances between the pSTA and the aDTA, pDTA, and MTA at the level of the zygomatic arch

\begin{tabular}{lccc}
\hline & & \multicolumn{2}{c}{ Distance, $\mathrm{mm}$} \\
\cline { 3 - 4 } & No. of Cases & Mean $(\mathrm{SD})$ & Range \\
\hline pSTA-aDTA & 18 & $50.3(4.65)$ & 42.0 to 60.4 \\
\hline pSTA-pDTA & 46 & $31.5(5.53)$ & 20.8 to 48.3 \\
\hline pSTA-MTA & 34 & $-1.68(4.48)$ & -13.5 to 7.8 \\
\hline
\end{tabular}


TABLE 6. Course of the MTA after branching from the STA

\begin{tabular}{lc}
\hline \multicolumn{1}{c}{ Course } & No. of Hemispheres $(n=34)$ \\
\hline Anteriorly & 0 \\
\hline Posterosuperiorly & 20 \\
\hline Superiorly & 14 \\
\hline
\end{tabular}

This result suggests that most of the MTAs were preserved in the posterior half of the muscle by CS and developed as collateral circulation.

In this study, the potential injury of collateral vessels by CS was also a concern. Because the incision line of CS was almost parallel to the course of the pSTA, we investigated its positional relationship to the aDTA, pDTA, and MTA. At the level of the zygomatic arch (the proximal end of CS), both the aDTA and pDTA were located far from the pSTA (mean distances 50.3 and $31.5 \mathrm{~mm}$ from the pSTA, respectively), whereas the MTA ran very close to the pSTA (mean distance $1.68 \mathrm{~mm}$ from the pSTA; Table 5). In addition, we also demonstrated that the MTA in all cases ran posteriorly or superiorly but not anteriorly (Table 6). Thus, CS parallel to the pSTA may injure the MTA at the proximal end; therefore, we propose that CS should be performed slightly anterior to the pSTA to avoid injury to the MTA.

\section{Limitations}

This study has several limitations. First, this study had a retrospective observational design. A prospective study is warranted to confirm the results. Second, we did not consider the influences of preoperative hemodynamic status and the effect of STA-MCA anastomosis on collateral developments. Because a complementary relationship has been reported between direct and indirect revascularization, ${ }^{1,13,20}$ quantitative assessment of these factors will provide more definitive conclusions. Third, we did not evaluate the contribution of other small arteries, such as the transverse facial, zygomatico-orbital, zygomaticotemporal, zygomatico-facial, and posterior auricular arteries, that supply blood to the temporal region..$^{18}$ SS and CS may have affected the collateral developments from these arteries.

\section{Conclusions}

SS has the potential of preventing postoperative neurological deterioration caused by the swollen temporalis muscle without inhibiting the development of the collateral vessels. Because the MTA courses close to the pSTA, particular attention needs to be paid during CS to avoid MTA injury.

\section{References}

1. Amin-Hanjani S, Singh A, Rifai H, Thulborn KR, Alaraj A, Aletich V, et al: Combined direct and indirect bypass for moyamoya: quantitative assessment of direct bypass flow over time. Neurosurgery 73:962-968, 2013

2. Bang JS, Kwon OK, Kim JE, Kang HS, Park H, Cho SY, et al: Quantitative angiographic comparison with the OSIRIS program between the direct and indirect revasculariza- tion modalities in adult moyamoya disease. Neurosurgery 70:625-633, 2012

3. Cheung LK: The vascular anatomy of the human temporalis muscle: implications for surgical splitting techniques. Int J Oral Maxillofac Surg 25:414-421, 1996

4. Czabanka M, Peña-Tapia P, Schubert GA, Woitzik J, Vajkoczy P, Schmiedek P: Characterization of cortical microvascularization in adult moyamoya disease. Stroke 39:1703-1709, 2008

5. Fujimura M, Kaneta T, Mugikura S, Shimizu H, Tominaga $\mathrm{T}$ : Temporary neurologic deterioration due to cerebral hyperperfusion after superficial temporal artery-middle cerebral artery anastomosis in patients with adult-onset moyamoya disease. Surg Neurol 67:273-282, 2007

6. Fujimura M, Kaneta T, Shimizu H, Tominaga T: Cerebral ischemia owing to compression of the brain by swollen temporal muscle used for encephalo-myo-synangiosis in moyamoya disease. Neurosurg Rev 32:245-249, 2009

7. Funaki T, Takahashi JC, Takagi Y, Kikuchi T, Yoshida K, Mitsuhara T, et al: Unstable moyamoya disease: clinical features and impact on perioperative ischemic complications. J Neurosurg 122:400-407, 2015

8. Imai H, Miyawaki S, Ono H, Nakatomi H, Yoshimoto Y, Saito N: The importance of encephalo-myo-synangiosis in surgical revascularization strategies for moyamoya disease in children and adults. World Neurosurg 83:691-699, 2015

9. Kanda Y: Investigation of the freely available easy-to-use software 'EZR' for medical statistics. Bone Marrow Transplant 48:452-458, 2013

10. Karasawa J, Kikuchi H, Furuse S, Sakaki T, Yoshida Y: A surgical treatment of "moyamoya" disease "encephalo-myo synangiosis". Neurol Med Chir (Tokyo) 17:29-37, 1977

11. Kazumata K, Ito M, Tokairin K, Ito Y, Houkin K, Nakayama $\mathrm{N}$, et al: The frequency of postoperative stroke in moyamoya disease following combined revascularization: a single-university series and systematic review. J Neurosurg 121:432440, 2014

12. Kobayashi K, Takeuchi S, Tsuchida T, Ito J: [Encephalo-myosynangiosis (EMS) in moyamoya disease - with special reference to postoperative angiography (author's transl).] Neurol Med Chir (Tokyo) 21:1229-1238, 1981 (Jpn)

13. Komura S, Mikami T, Sugino T, Suzuki Y, Komatsu K, Wanibuchi M, et al: Complementary relation between direct and indirect bypass in progress of collateral circulation in moyamoya disease. World Neurosurg 104:197-204, 2017

14. Kuroda S, Houkin K: Moyamoya disease: current concepts and future perspectives. Lancet Neurol 7:1056-1066, 2008

15. Machida T, Nakano S, Ishige S, Ono J, Fujikawa A: Subcortical low-intensity lesions on fluid-attenuated inversion recovery images after revascularization surgery for moyamoya disease. World Neurosurg 98:512-519, 2017

16. Miyamoto S, Yoshimoto T, Hashimoto N, Okada Y, Tsuji I, Tominaga T, et al: Effects of extracranial-intracranial bypass for patients with hemorrhagic moyamoya disease: results of the Japan Adult Moyamoya Trial. Stroke 45:1415-1421, 2014

17. Mukerji N, Cook DJ, Steinberg GK: Is local hypoperfusion the reason for transient neurological deficits after STA-MCA bypass for moyamoya disease? J Neurosurg 122:90-94, 2015

18. Nakajima H, Imanishi N, Minabe T: The arterial anatomy of the temporal region and the vascular basis of various temporal flaps. Br J Plast Surg 48:439-450, 1995

19. Takemura S, Sato S, Kuroki A, Saito S, Kayama T: [New ideas for indirect revascularization surgery for moyamoya disease.] No Shinkei Geka 27:987-992, 1999 (Jpn)

20. Uchino H, Kim JH, Fujima N, Kazumata K, Ito M, Nakayama N, et al: Synergistic interactions between direct and indirect bypasses in combined procedures: the significance 
of indirect bypasses in moyamoya disease. Neurosurgery 80:201-209, 2017

\section{Disclosures}

The authors report no conflict of interest concerning the materials or methods used in this study or the findings specified in this paper.

\section{Author Contributions}

Conception and design: Machida. Acquisition of data: all authors. Analysis and interpretation of data: Machida, Higuchi, Ishige. Drafting the article: Machida, Higuchi, Ono. Critically revising the article: Machida, Higuchi, Ono. Reviewed submitted version of manuscript: Machida, Higuchi, Ono. Approved the final version of the manuscript on behalf of all authors: Machida. Statistical analysis: Machida. Study supervision: Higuchi, Ono.

\section{Supplemental Information}

\section{Previous Presentations}

Portions of this work were reported in abstract and oral presentation forms at the 41st annual meeting of the Japanese Society of Surgery for Cerebral Stroke, Fukuoka, Japan, April 26, 2012.

\section{Correspondence}

Toshio Machida: Chiba Cerebral and Cardiovascular Center, Chiba, Japan.tmachida-nsu@umin.ac.jp. 